\title{
Leishmania major: Parasite Interactions Suggesting Sexuality
}

\author{
Maria Auxiliadora de Sousa/ ${ }^{+}$, Mirian Claudia de Souza Pereira*, \\ Suzana Côrte-Real*
}

\author{
Coleção de Tripanosomatídeos, Departamento de Protozoologia *Departamento de Ultraestrutura e Biologia \\ Celular, Instituto Oswaldo Cruz, Av. Brasil 4365, 21045-900 Rio de Janeiro, RJ, Brasil
}

In five experiments, Leishmania (Leishmania) major (MRHO/SU/59/P-strain) grew poorly when seeded in FYTS medium supplemented with $15 \%$ fetal calf serum, but presented several peculiar pairs of promastigotes diametrically opposed and attached at their posterior ends (5.8-13.5\%). As seen in Giemsa-stained smears, a ring-like line and/or an enlargement, generally occurred at the parasite junction. A close proximity of nuclei, which sometimes were difficult to distinguish from each other, was also observed at this junction. Several of these pairs appeared to be composed of fused cells in which the nuclei could be apparently fused, as shown by fluorescence microscopy to detect $\beta$-tubulin and DNA, and by scanning electron microscopy. Under other culture conditions these pairs were absent or occurred at very low rates (0.2-2.2\%). Such pairs differ markedly from longitudinally dividing cells and resemble those described in two other Leishmania species, as well as in Herpetomonas megaseliae and Phytomonas davidi, suggesting steps of a putative sexual process.

Key words: Trypanosomatidae - Leishmania major - sexuality - morphology -immunocytochemistry - scanning electron microscopy

The occurrence of sexuality in Leishmania has been a subject of interest since the beginning of this century, but several older cytological observations ascribed to sexual events were unconvincing (Rogers 1904, Adie 1921-22, Christophers et al. 1926, Wenyon 1926, Wenrich 1954). This subject was discredited, until Maazoun et al. (1981) reported heterozygous patterns in enzyme electrophoretic variants of three Leishmania species, and their paper was followed by several others describing naturally occurring putative hybrids using biochemical and/or molecular methods (Le Blancq et al. 1983, Le Blancq \& Peters 1986, Evans et al. 1987, Pagès et al. 1989, Kelly et al. 1991, Cupolillo et al. 1992, Belli et al. 1994, Piarroux et al. 1994, Dujardin et al. 1995). However, genetic recombination between species or strains of Leishmania has not yet been demonstrated under laboratory conditions (Gradoni et al. 1986, Evans et al. 1989, Panton et al. 1991, Shehata et al. 1991), although Lanotte and Rioux (1990) had recorded by videocinematography fusion of pairs of apposed promastigotes in cultures of two species, and also evidenced the possibility of nuclear fusion by examining these cultures in Giemsa-stained smears.

In this paper we describe pairs of apposed promastigotes occurring at relatively high percent

\footnotetext{
${ }^{+}$Corresponding author. Fax: $+55-21-260.4434$ or 290.9339

Received 30 December 1996

Accepted 10 July 1997
}

in $L$. (L.) major (P strain) when seeded in a medium described for insect trypanosomatids (Roitman et al. 1972), which we had supplemented with fetal calf serum. Such pairs were studied on Giemsa-stained smears, by fluorescence microscopy to examine the B-tubulin distribution and nuclear DNA features, as well as by scanning electron microscopy. The frequency of such pairs under other culture conditions was also examined.

\section{MATERIALS AND METHODS}

Promastigotes of a L. (L.) major strain (MRHO/ $\mathrm{SU} / 59 / \mathrm{P}$ ) grown in BHI+LIT medium overlaying blood-agar (Jaffe et al. 1984) were seeded in FYTS medium (Roitman et al. 1972) supplemented with $15 \%$ heat-inactivated fetal calf serum (FYTS+ $15 \% \mathrm{FCS}$ ), $\mathrm{pH} 7.0$, which was distributed in 4-mlvolumes in 16x150 mm screwcap tubes, and kept at about $27^{\circ} \mathrm{C}$. Following the finding in fresh preparations of unusual pairs of apposed cells, Giemsastained smears were prepared as previously described (Sousa 1994) for morphological studies. Subsequently, two experiments were performed to compare the frequency of such pairs in FYTS $+15 \%$ FCS with those in LIT medium (Chiari \& Camargo 1984), LIT/blood-agar and FYTS+ $15 \%$ FCS/blood-agar. The inoculum was always promastigotes from BHI+LIT/blood-agar cultures, which in the first experiment had no pair of apposed cells, while in the second one presented $0.5 \%$. The cultures were maintained at about $27^{\circ} \mathrm{C}$ and the percent of pairs of apposed cells was determined daily, from the 2 nd to the 7 th days, by 
examining at least 500 cells in fresh preparations. The maximum parasite growth was compared in two culture conditions, FYTS+15\%FCS and LIT/ blood-agar, in both cases beginning with about $8.5 \times 10^{5}$ cells $/ \mathrm{ml}$. The occurrence of pairs of apposed cells in FYTS+15\%FCS was also verified by using promastigotes from LIT/blood-agar as inoculum (3 assays).

Pairs of apposed cells from a 5-day old culture in FYTS+15\%FCS were studied under fluorescence microscopy to examine concomitantly the B-tubulin distribution and the nuclear DNA. Then, the cells were washed in phosphate buffered saline (PBS), $\mathrm{pH} 7.2$, allowed to adhere to $0.1 \%$ polyL-lysine-coated coverslips, fixed for $5 \mathrm{~min}$ at room temperature with $4 \%$ paraformaldehyde in PBS and rinsed three times in PBS containing 0.5\% Triton $\mathrm{X}-100$. Subsequently, the cells were incubated for $1 \mathrm{hr}$ at $37^{\circ} \mathrm{C}$ with 1:50 anti- $\beta$ tubulin monoclonal antibody (Sigma), rinsed and incubated for $1 \mathrm{hr}$ with 1:25 fluorescein isothiocyanate (FITC)coupled rabbit anti-mouse IgG; following a $10 \mathrm{~min}$ incubation with $2 \mathrm{mg} / \mathrm{ml}$ 4,6-diamidino-2phenylindole (DAPI; Sigma), the coverslips were rinsed in $0.85 \% \mathrm{NaCl}$ and mounted in 1.4diazabicyclo-(2.2.2)-octane (DABCO; Sigma). The preparations were observed and photographed with a Zeiss microscope equipped for epifluorescence using selective FITC or DAPI filters.

For scanning electron microscopy, cells from a 5-day old culture in FYTS+15\%FCS were adhered to poly-L-lysine coated coverslips as above-described, fixed for $1 \mathrm{hr}$ at $4^{\circ} \mathrm{C}$ in $2.5 \%$ glutaraldehyde in $0.1 \mathrm{M} \mathrm{Na}$-cacodylate buffer, $\mathrm{pH} 7.2$, plus $3.5 \%$ sucrose, and subsequently washed in this same buffer. After post-fixation in $1 \% \mathrm{OsO}_{4}$ for $30 \mathrm{~min}$, the material was dehydrated in graded series of acetone, dried with $\mathrm{CO}_{2}$ by the critical-point method, mounted on aluminum stubs and coated with a $20 \mathrm{~nm}$-thick gold layer. The samples were examined with a Carl Zeiss DSM 940 scanning electron microscope.

\section{RESULTS}

FYTS+15\%FCS medium was unsuitable for growing $L$. major promastigotes, whose population increased somewhat more than twice within 48-72 $\mathrm{hr}$, but thereafter gradually declined. However, such cultures presented a relatively high percent (5.8$13.5 \%$ ) of peculiar pairs of promastigotes, at times very slender, diametrically opposed to each other and attached at their posterior ends, there generally occurring a ring-like line and/or an enlargement (Figs 1-9) sometimes resembling a hat brim (Fig.

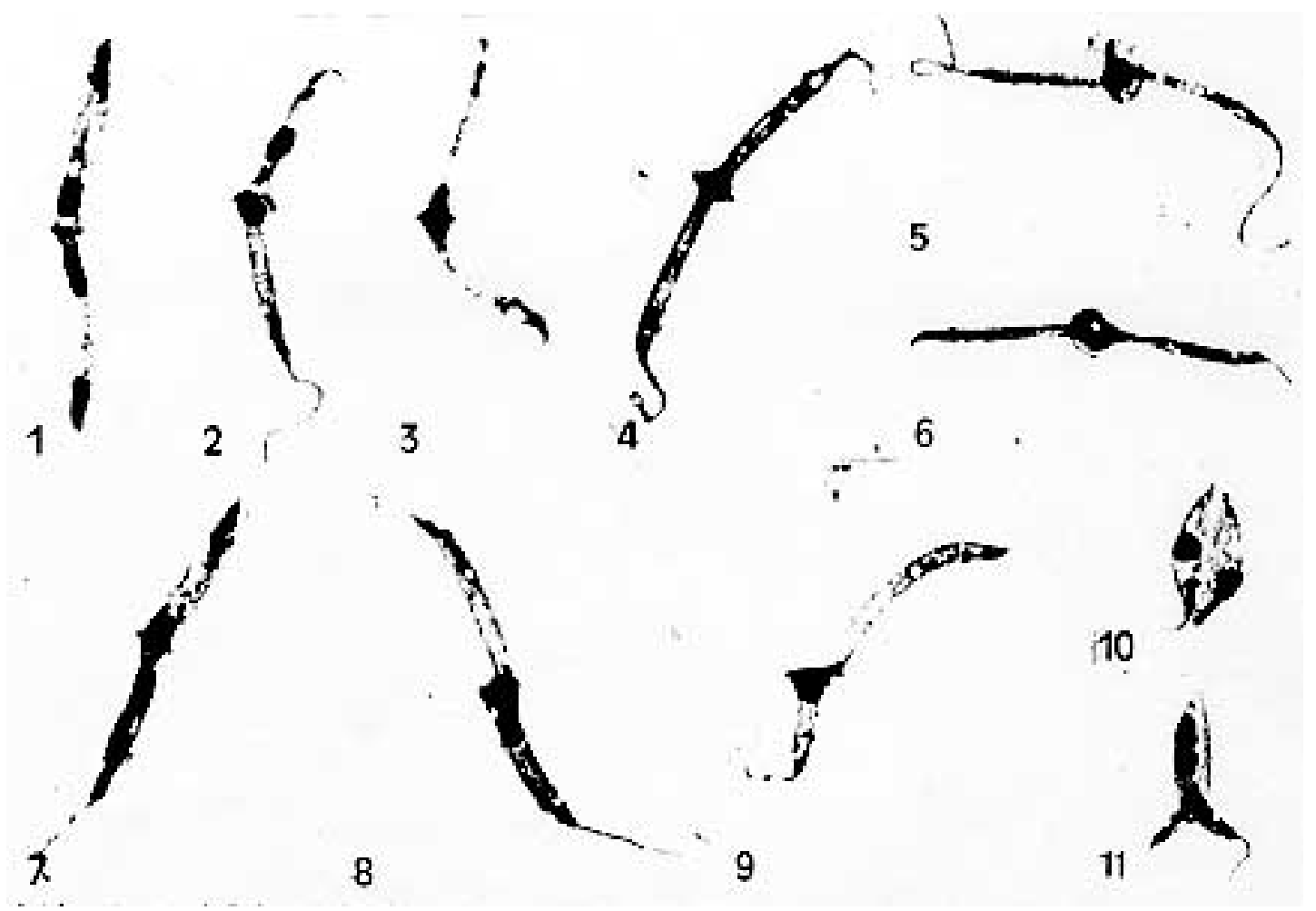

Leishmania major in Roitman's FYTS medium supplemented with $15 \%$ heat-inactivated fetal calf serum. Giemsa stain after $\mathrm{HCl}$ treatment. X 1,500. Figs 1-9: peculiar pairs of promastigotes suggesting migration and fusion of nuclei; note the ring-like line and/ or enlargement at the cell junction, which in Fig. 5 resembles a hat brim. Figs 10-11: longitudinally dividing parasites. 
5), as seen in Giemsa-stained smears. In such pairs both nuclei were usually in close proximity and in some instances it was difficult to distinguish one from the other, either in Giemsa-stained slides or under DAPI fluorescence (Figs 3-6, 9, 12B-14B, $15,16)$. Several pairs seemed composed of fused cells, as suggested either by indirect immunofluorescence of B-tubulin (Figs 12A, 13A, arrows) or by scanning electron microscopy, which also evidenced the peculiar enlargement and meeting of nuclei at the level of the parasite junction (Figs 1721). Typically dividing cells could also be seen in FYTS+15\%FCS (Figs 10, 11).

In fresh preparations, the pairs under study were actively moving and presented an uncommon brightness at the level of nucleus. The phenomenon of formation of these pairs in FYTS+15\% FCS medium was reproducible in 5/5 assays, the inoculum being promastigotes either from BHI+LIT/ blood-agar or LIT/blood-agar. The percent of these pairs in different culture conditions are given in Table. L. major grew luxuriantly in LIT/blood-agar and to a lesser extent in other media (FYTS $+15 \%$ FCS/blood-agar $>$ LIT $>$ FYTS + $15 \%$ FCS). Beginning with about $8.5 \times 10^{5}$ promastigotes $/ \mathrm{ml}$, the maximum growth in LIT/ blood-agar was nearly $6 \times 10^{7}$ cells $/ \mathrm{ml}$ (5th day),

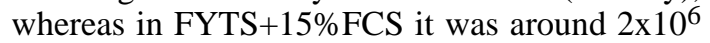
cells/ml (2nd day).

\section{DISCUSSION}

To our knowledge, in Leishmania species, pairs of promastigotes such as these described herein have not been reported previously. They also differ from those found in another L. major strain during experimental infection in Lutzomyia longipalpis (Walters et al. 1993), which were apposed paramastigotes. These pairs also markedly differ from cells joined laterally during longitudinal binary fission (compare Figs 1-9 with 10 and 11). It is worthy mentioning that although dividing trypanosomatids can occasionally be attached by their posterior ends before complete separation, they neither present any enlargement nor the proximity of nuclei at the level of their junction, as commonly seen here. However, the pairs of promastigotes described by us are somewhat similar to those reported in Herpetomonas megaseliae and Phytomonas davidi (Sousa 1991, 1994), and resemble those formed by fusion previously described in $L$. infantum and L. tropica (Lanotte \& Rioux 1990), since the parasite attachment occurs by the posterior ends, the cells remaining apposed to each other, and on several occasions nuclear fusion appears to take place. Our studies to detect both B-tubulin and DNA in such pairs, as well as our findings by scanning electron microscopy,

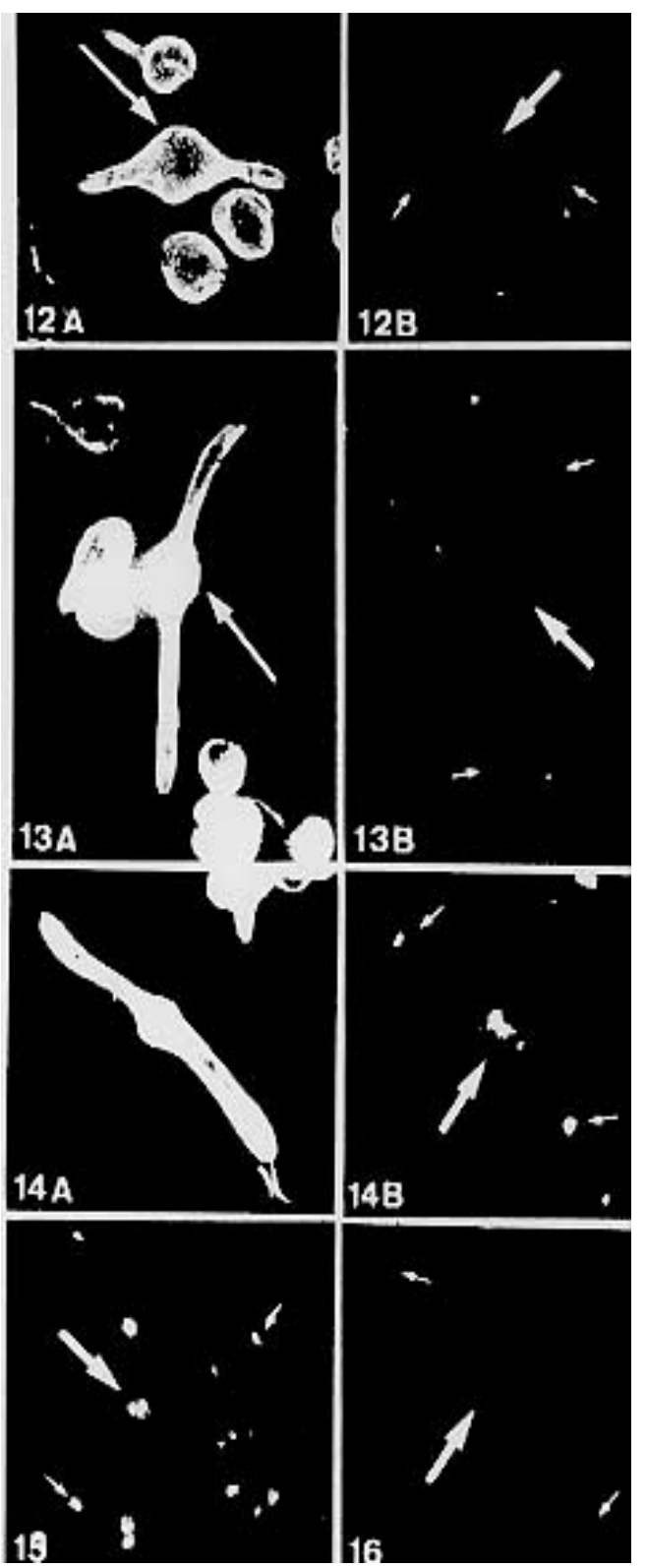

Leishmania major in Roitman's FYTS medium supplemented with $15 \%$ heat-inactivated fetal calf serum. Fluorescence microscopy of doubly labeled cells to detect $\beta$-tubulin and DNA. $X$ 1,500. Figs 12A-14A: indirect immunofluorescence of tubulin in pairs of apposed cells showing no discontinuity at their junction in Figs 12A and 13A (arrows). Figs 12B-14B, 15-16: DAPI fluorescence of kinetoplast (short arrow) and nucleus (large arrow) in pairs of apposed cells; note the very close proximity of nuclei, mainly in Figs 15 and 16.

strongly suggest cellular fusion in the majority of cases, as well as close contact or even fusion of the nuclei (Figs 12A-21). Taking together our findings and those from the literature, we have considered the possibility that a fusion process to pro- 


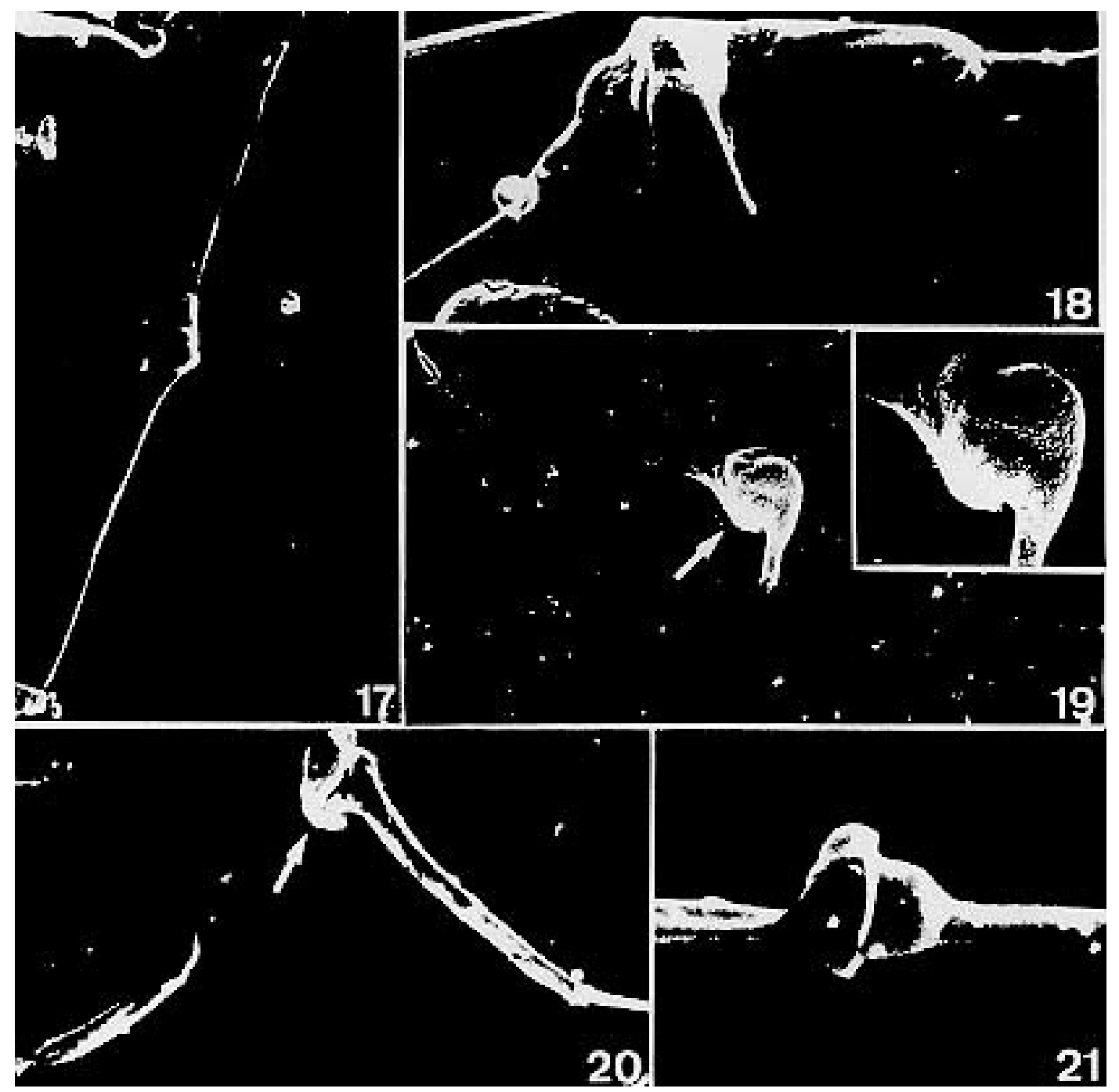

Leishmania major in Roitman's FYTS medium supplemented with 15\% heat-inactivated fetal calf serum. Scanning electron microscopy. Fig. 17: pair of very slender cells, diametrically opposed to each other, and presenting the nuclei at the level of their junction (X 3,100). Fig. 18: this pair illustrates the possibility of cellular fusion (X 7,000). Fig. 19: pair of very slender cells fused at their posterior ends, there occurring close contact of nuclei, arrow (X 3,340; X 6,500). Fig. 20: note the peculiar hat brim-like enlargement (arrow) at the parasite junction (X 3,600). Fig.21: nuclear meeting at the cellular junction (X 6,750).

mote nuclear interactions could have generated the pairs here described, this strongly suggesting a sexual process. Our data confirm that in trypanosomatids phenomena can occur in which the cytological bases for genetic recombination are clearly present.

The finding of putative hybrids of other $L$. major isolates (Le Blancq et al. 1983) and of $L$. major/L. arabica (Evans et al. 1987, Kelly et al. 1991) support the hypothesis of occurrence of genetic exchange in these species, although it has been considered infrequent (Le Blancq et al. 1983, Kelly et al. 1991). In the present study, we verified that the pairs of apposed promastigotes suggesting a sexual process were not common in media other than FYTS+15\%FCS, they usually not being found or occurring in low numbers. It is accepted that genetic recombination mediated by sex is generally a major mechanism promoting diversity within a species, consequently enhancing its chances of survival in a fluctuating environment. As our paper shows close association between two cells and their nuclei, the question arises whether the unsuitable conditions for L. major growth in supplemented FYTS medium could have triggered the formation of such pairs to propitiate some type of 
TABLE

Percent of pairs of apposed promastigotes in Leishmania major under different culture conditions; parasites from $\mathrm{BHI}+\mathrm{LIT} / \mathrm{blood}$-agar were used as inoculum

\begin{tabular}{|c|c|c|c|c|c|c|}
\hline \multirow{2}{*}{ Days } & \multicolumn{2}{|c|}{ FYTS+15\%FCS } & \multirow[t]{2}{*}{ LIT } & \multicolumn{2}{|c|}{ LIT/blood-agar } & \multirow{2}{*}{$\begin{array}{c}\text { FYTS+15\%FCS } \\
\text { blood agar }\end{array}$} \\
\hline & (1) & $(2)$ & & (1) & (2) & \\
\hline 2 & 13.0 & 7.3 & 0 & 0.3 & 0 & 0.7 \\
\hline 3 & 11.5 & 13.5 & 2.0 & 0.9 & 0 & 1.2 \\
\hline 4 & 7.7 & 5.8 & 0.3 & 1.4 & 0 & 1.1 \\
\hline 5 & 8.5 & 5.7 & 2.2 & 0.2 & 0.2 & 0.9 \\
\hline 6 & 7.8 & 5.8 & 0.8 & 0.6 & 0 & 0.8 \\
\hline 7 & 5.8 & 6.3 & 0 & 0.2 & 0 & 1.4 \\
\hline
\end{tabular}

(1) and (2), respectively, the 1 st and 2 nd experiments.

BHI: brain heart infusion medium; LIT: liver infusion-tryptose medium; FYTS+15\%FCS: folic acid-yeast extracttryptose-sucrose medium supplemented with $15 \%$ fetal calf serum.

genetic exchange. There is much to be done on this subject and studies are planned to explain better the present findings, such as determining the biochemical and molecular structure of this organism, both before and after being cultured in supplemented FYTS.

\section{ACKNOWLEDGMENTS}

To Dr Wladimir Lobato Paraense for reviewing the manuscript, Miss Sheila Medeiros dos Santos for technical assistance and Dr Monika Barth for allowing several photomicrographs to be taken at her laboratory.

\section{REFERENCES}

Adie HA 1921-22. Preliminary note on the development of the Leishman donovan parasite in spleen juice and in the alimentary tract of Cimex lectularius (Lin.). Indian J Med Res 9: 255-260.

Belli AA, Miles MA, Kelly JM 1994. A putative Leishmania panamensis/Leishmania braziliensis hybrid is a causative agent of human cutaneous leishmaniasis in Nicaragua. Parasitology 109: 435-442.

Chiari E, Camargo EP 1984. Culturing and cloning of Trypanosoma cruzi, p. 23-26. In CM Morel, Genes and Antigens of Parasites. A Laboratory Manual, Fundação Oswaldo Cruz, Rio de Janeiro.

Christophers SR, Shortt HE, Barraud PJ 1926. The morphology and life cycle of the parasite of Indian kalazar in culture. Indian Med Res Mem 4: 19-53.

Cupolillo E, Grimaldi Jr G, Momen H 1992. Hybrid parasites in the Leishmania braziliensis "complex" causing cutaneous leishmaniasis in humans. Isozyme Bull 25: 51.

Dujardin J-C, Bañuls A-L, Llanos-Cuentas A, Alvarez E, DeDoncker S, Jacquet D, Le Ray D, Arevalo J, Tibayrenc M 1995. Putative Leishmania hybrids in the Eastern Andean valley of Huanuco, Peru. Acta Tropica 59: 293-307.

Evans DA, Kennedy WPK, Elbihari S, Chapman CJ, Smith V, Peters W 1987. Hybrid formation within the genus Leishmania? Parassitologia 29: 165-173.

Evans DA, Smith V, Killick-Kendrick R, Neal RA, Peters W 1989. Evidence for hybrid formation in the genus Leishmania, p. 685-690. In DT Hart, Leishmaniasis: the current status and new strategies for control, Plenum Press, New York, London.

Gradoni L, Gramiccia M, Maroli M, Pozio E 1986. Infestations mixtes de Phlebotomus perniciosus Newstead, 1911 par différents zymodèmes du complexe Leishmania infantum. Etude expérimentale, p. 305-310. In J-A Rioux, Leishmania: Taxonomie et Phylogenèse. Applications écoépidémiologiques, IMEEE, Montpellier.

Jaffe CL, Grimaldi G, McMahon-Pratt D 1984. The cultivation and cloning of Leishmania, p. 47-91. In CM Morel, Genes and Antigens of Parasites. A Laboratory Manual, Fundação Oswaldo Cruz, Rio de Janeiro.

Kelly JM, Law JM, Chapman CJ, Van Eys GJJM, Evans DA 1991. Evidence of genetic recombination in Leishmania. Mol Biochem Parasitol 46: 253-264.

Lanotte G, Rioux J-A 1990. Fusion cellulaire chez les Leishmania (Kinetoplastida, Trypanosomatidae). C $R$ Acad Sci Paris 310 (série III): 285-288.

Le Blancq SM, Peters W 1986. Leishmania in the Old World: 2. Heterogeneity among L. tropica zymodemes. Trans R Soc Trop Med Hyg 80: 113119.

Le Blancq SM, Schnur LF, Schlein Y 1983. An apparent association of enzymic variants of Leishmania major with specific geographical areas in Israel. Bull Soc Path Exot 76: 543-548.

Maazoun R, Lanotte G, Rioux J-A, Pasteur N, KillickKendrick R, Pratlong F 1981. Signification du polymorphisme enzymatique chez les Leishmanies. Ann Parasitol Hum Comp 56: 467-475.

Pagès M, Bastien P, Veas F, Rossi V, Bellis M, Wincker P, Rioux J-A, Roizès G 1989. Chromosome size and number polymorphisms in Leishmania infantum suggest amplification/deletion and possible genetic exchange. Mol Biochem Parasitol 36: 161-168.

Panton LJ, Tesh RB, Nadeau KC, Beverley SM 1991. A test for genetic exchange in mixed infections of Leishmania major in the sand fly Phlebotomus papatasi. J Protozool 38: 224-228.

Piarroux R, Trouvé V, Pratlong F, Martini A., Lambert 
M, Rioux JA 1994. The use of isoelectric focusing on polyacrilamyde gel for enzymatic analysis of "Old World" Leishmania species. Trans R Soc Trop Med Hyg 88: 475-478.

Rogers L 1904. On the development of flagellated organism (trypanosomes) from the spleen protozoic parasites of cachexial fevers and kala-azar. $Q J$ Microsc Sci 48: 367-377.

Roitman C, Roitman I, Azevedo HP 1972. Growth of an insect trypanosomatid at $37^{\circ} \mathrm{C}$ in a defined medium. J Protozool 19: 346-349.

Shehata M, El Sattar S, Morsy T, El Sawaf B 1991. Experimental dual infection of Leishmania in Phlebotomus langeroni (Diptera: Psychodidae). Trans $R$ Soc Trop Med Hyg 85: 739-740.

Sousa MA 1991. Cytological events suggesting sexuality in a trypanosomatid. Mem Inst Oswaldo Cruz 86
(Suppl 1): 268

Sousa MA 1994. Cell-to-cell interactions suggesting a sexual process in Herpetomonas megaseliae (Kinetoplastida: Trypanosomatidae). Parasitol Res 80: 112-116.

Walters LL, Irons KP, Chaplin G, Tesh RB 1993. Life cycle of Leishmania major (Kinetoplastida: Trypanosomatidae) in the neotropical sand fly Lutzomyia longipalpis (Diptera: Psychodidae). J Med Entomol 30: 699-718.

Wenrich DH 1954. Sex in Protozoa. A comparative review, p. 134-265. In DH Wenrich, IF Lewis, JR Raper (eds), Sex in Microorganisms, American Association for Advancement of Science, Washington.

Wenyon CM 1926. Protozoology. A Manual for Medical Men, Veterinarian and Zoologists, vol I, p. 336340, Baillière, Tindall and Cox, London. 